\title{
Introduction to the Network Analysis of Digital and Social Media Minitrack
}

\author{
Devan Rosen \\ Ithaca College \\ drosen@ithaca.edu
}

\author{
George A. Barnett \\ University of California - Davis \\ gbarnett@ucdavis.edu
}

\author{
Kar-Hai Chu \\ University of Pittsburgh \\ chuk@pitt.edu
}

\section{Introduction}

Social networks are representation of complex systems defined by the patterns of relationships among a system's components. Networks are created by information flows or other relations among entities through time and space. The goal of network analysis is to describe the structure of a higher-level system based on the pattern of linkages among a set of lower level nodes, and how this structure changes over time. It differs from traditional research by focusing on relationships rather than the attributes of individuals. The complex social networks that people create and manage are dynamic, multi-modal, and increasingly mediated by social and digital media.

Digital and social media have provided precise measures of social networks. From social networking sites (e.g. Facebook), to microbloging networks (e.g. Twitter) and global cooperation networks (e.g. CouchSurfing), the material and nonmaterial exchanges of individuals and larger social systems are being recorded and largely retrievable, presenting social network researchers with powerful new data.

There are four main implications of having access to digital and social media data. First, we now have access to measurements of network relations that are more reliable than in the past. Second, the nature of the relational data is unique, allowing for the analytical exploration of network structures in ways that are pushing the boundaries of the science. Third, analysis of network evolution is increasingly possible since much of the data available through social media is dynamic (a historically persistent challenge when collecting network data). Fourth, dynamic network data has contributed to visualization methods that allow for the animation of social networks.

Network science has progressed in parallel with the development of social and digital media, computers and other information systems. The increased theoretical understanding and analytic representation of computer and information systems provides developers with a greater sense of how people and organizations utilize technology to manage resources embedded in their social and digital networks.

The papers in this minitrack represent theoretical and analytic developments in social and digital media research. They focus on new media and information technology, including organizational networks, blogs, Twitter, and collaboration networks. They cross disciplines and levels of analysis, using novel approaches to discover aspects of digital networks.

\section{Papers included in the minitrack}

In The Effect of Turnover Intention on Tie Formation in Online Organization Networks, Wallace Chipidza and John Tripp investigate the relationship between organizational turnover and communication relationships. Their hypotheses are tested using exponential random graph modeling on the email and advice networks of a technology company. In attempting to identify individuals that could potentially leave a company, they do not focus solely on centrality measures but on the existing relationships within the networks. Impacts of the research include contributions to organizational research that could lead to interventions to prevent or reduce turnover.

In Bucking the Trend: An Agentive Perspective of Managerial Influence on Blog's Attractiveness, Carlos Denner dos Santos, Isadora Castro, George Kuk, Silvia Onoyama, and Marina Moreira look into the attractiveness of blogs. However, rather than a traditional examination of environmental influence, the authors use social network theory and organizational theory to study how bloggers adapt their behaviors based on their structural network positions. Their agentive perspective was able to find that bloggers who are mindful of the content of others in their network can leverage that information to attract more traffic to their blogs. It is a unique study and provides implications for practice for both new and veteran bloggers.

In Global Contagion of Non-Viral Information, Alon Bartal, Gilad Ravid, and Oren Tsur present research that disambiguates viral and non-viral 
information diffusion. They explore how non-viral contagion occurs on Twitter, both locally through follower retweets as well as globally from nonfollowers. The results show that while close proximately to the original tweet increases the likelihood of local contagion, global exposure can also increase the chance of retweets for individuals that are only remotely connected to the original source of information. The authors also found that topic areas differ between non-viral global and local contagion, suggesting it may be important for researchers to consider both forms of contagion when analyzing the spread of information across online networks.

In Does Our World “weigh” Less Right Now? The Gravitational Pull in a Scientific Collaboration Network is Getting Weaker with Time, Guy Kelman, Moshe Levy, and Eran Manes suggest that the deployment of the internet and social media systems, reduced airfares and other marks of progress in communication have made distance collaboration easier, resulting in increases in geography distance among collaborators in scientific research. They explore letters and articles between coauthors and find that the probability density function of coauthorship is linearly related to the distance between authors, noted by a separation before and after the advent of the internet. The authors apply gravitational laws, rather than sociological frameworks, to understand the network environment, and calculate a gravitational constant to show the increased ease with which distances are now crossed. 